\title{
CLINICAL AND RADIOGRAPHIC EVALUATION OF THE USE OF INTRAORAL IMPLANTS IN CONSTRUCTON OF SINGLE STAGE IMPLANT RETAINED AURICULAR PROSTHESES
}

\author{
Ingy S. Soliman ${ }^{1} M s c$, Mohamed S. El-Attar ${ }^{2} P h D$, Mohamed M. Fata ${ }^{3} P h D$, Juan R. Garcia \\ ${ }^{4} \mathrm{PhD}$, Osama Abdel Aziz ${ }^{5} P h D$, Ahmed Y. Kosba ${ }^{6}$ Msc, Maha El-Demellawy ${ }^{7} P h D$. \\ ABSTRACT
}

INTRODUCTION: Although plastic surgery is capable of restoring missing tissues, it may not be the ideal choice of treatment because of the complex shape and structure. For restoration of complex organs such as ears, prosthetic restorations may be an alternative to surgical restorations due to their aesthetical success. Prosthetic rehabilitation to restore the facial disfigurements with custom made prosthetic devices may improve the level of function and patient's self-esteem. Difficulties with facial prostheses may arise due to patient factors such as amount of soft tissue loss, location of the auricular defect, dynamic tissue beds, retentive quality of the area, and associated irritation of the tissue beds. Hence, the rehabilitation choice depends on meticulous restoration of physical dimensions, external contour, and surface landmarks to ensure satisfactory aesthetic outcomes for prosthodontists and their patients.

OBJECTIVES: To use inrta-oral implant as an alternate of maxillofacial ones to retain auricular prostheses and evaluate autografted single stage implant placement surgery.

MATERIALS AND METHODS: Ten patients complaining of congenitally missing ear either microtia or anotia or acquired missing ones were recruited from maxillofacial and prosthodontic departments' outpatient clinics, Faculty of dentistry, Alexandria University. Patients were diagnosed clinically and radio-graphically using Cone Beam Computed tomography imaging (CBCT). The prosthetic ear was retained using three ball and socket attachments.They were evaluated upon radiographic and clinical level of osseointegration as well as patient acceptance and hygiene.

RESULTS: None of the implants showed failure to osseointegration; providing a survival rate of $100 \%$ on both clinical and radiographic levels of evaluation.

CONCLUSIONS: The use of $6 \mathrm{~mm}$ dental implants in the mastoid region succeeded to replace the maxillofacial implants. Single stage implant placement surgery minimized tissue traumatization and possessed higher patient satisfaction level. KEYWORDS: Intraoral implants, single stage surgery, auricular prosthesis, Autografted implants.

1. Assistant lecturer, Removable Prosthodontics Department, Faculty of Dentistry, Alexandria University.

2. Professor of Removable Prosthodontics Department, Faculty of Dentistry, Alexandria University, Clinical associate professor of prothodontics Department, University of Pittsburg.

3. Professor of Plastic and Maxillofacial Surgery Department, Faculty of Dentistry, Alexandria University.

4. Associate Professor,Department of Art as applied to Medicine, John Hopkins University, Certified Clinical Anaplastologist, Clinic Director, 3D Print Lab, Carnegie Center for Surgical Innovation.

5. Professor of Removable Prosthodontics Department, Faculty of Dentistry, Alexandria University

6. Assistant lecturer, Plastic and Maxillofacial Surgery Department, Faculty of Dentistry, Alexandria University.

7. Professor, Medical Biotechnology Department, genetic Engineering and Biotechnology Research Institute, City for research and Technology Applications.

\section{INTRODUCTION}

The rehabilitation of patients with head and neck disability due to either congenital or acquired defect is a challenging task. It is a difficult task because the defects are large and the soft tissues may be present in a healing Condition. Maxillofacial prosthesis need to fulfill two essential requirements, esthetics and function (1).

The last few decades have witnessed a significant increase in extensive malignancies of the head and neck region(2). This has resulted in increasing number of patients with extensive post-surgical defects. Many of them need to be suitably rehabilitated to minimize long term physical, functional and psychological consequences and ensure early return to normal life. In addition, these patients could be more willing to accept large surgical resections, if counseled about prosthetic reconstruction, prior to definitive surgery. It is crucial that all such patients receive a pre-operative referral to a maxillofacial prosthodontist prior to surgery (3).

Microtia is the most well known congenital anomaly of the auricle, and it results from incomplete embryonic development that is described as a congenital small and malformed external ear. The deformtiy occurs with a frequency of one in every 7000 to 8000 live births (4). The prevalence is significantly higher in Hispanics, Asians, Native Americans and Andeans. Males are affected more than females, and most cases of microtia are unilateral with a higher rate of occurrence on the right side. Environmental and genetic factors are important in the etiology of microtia (5). Microtia can cause psychological morbidity and surgical repair results in significant relief (6).

Classification systems have been developed to facilitate diagnosis, surgical repair and research studies of microtia. Hermann Marx described the first classification system in 1926, which was later modified by Meurman. Marx/Meurman(7,8) classified microtia into four degrees based on the vestigial remnant. In grade I microtia, all of the structures are present but with variable degrees of hypoplasia of the auricle, with cupping and variable external auditory stenosis. In grade II, variable hypoplasia of the concha is often accompanied by the absence of the external auditory canal. Grade III is the classic 'peanut ear', 
in which the auricle is absent and the lobule has an abnormal shape and position. Grade IV, known as anotia, is the most severe form of microtia, which is characterized by the complete absence of external ear (8).

Despite the advances in other methods, autologous costal cartilage graft remains the mainstream of ear reconstruction surgery. The acceptable aesthetic results and durability of the cartilaginous framework in long-term follow-up have contributed to the success of this method (9). The Tanzer (10), Walton and Beahm (9) and Brent (11) methods have provided the basis for current autologous reconstruction techniques. Historically, it has required three or four stages to create an ear. Current techniques have evolved to reduce the number of required stages.

It is extremely important that the surgeon attempting to reconstruct the auricle fully comprehends its threedimensional [3-D] morphologic features. The best material for fabrication of the 3-D framework is autogenous costal cartilage because of the least number of postoperative complications. The criteria for auricular reconstruction are: the patient is to be over 10-years-old; and the chest circumference at the level of the xiphoid process is to be at least $60 \mathrm{~cm}$. The first stage operation is the fabrication and grafting of the three-dimensional costal cartilage framework [3-D frame] and the second stage operation is the projection of the reconstructed auricle. It is important to comprehend the relationship between the angle of projection, distance from the temporal surface to the highest plane of the auricle and the height of the cartilage construct for a successful and symmetrical projection of the reconstructed auricle. With the use of the temproparietal fascia flap [TPF] and ultra-delicate split thickness scalp skin [UDSTS] during the first stage operation, the problems created by low hairline have been resolved, and the surgical results have been consistent and satisfactory. Skin grafting alone for auricular reconstruction is contraindicated (9-11).

Medpor (Stryker, USA) is a synthetic biocompatible porous polyethylene implant. Reinisch (12) has pioneered its use as an alternative to conventional autologous rib cartilage graft for ear reconstruction. Through the past several decades, he has made several modifications to both the implant and his surgical technique to decrease the complications of this method.

The ear prosthesis or epithesis is an alternative to plastic surgery. There are several methods for retention of prosthesis. However, the osseointegration described first by Brånemark (13) during the 1950s has become the most reliable and durable method for fixation of the prosthesis. Using titanium implants to integrate facial or cranial prostheses into living bone has been proven to be safe and is associated with predictable esthetic results (13).

Maxillofacial rehabilitation is advantageous for such patients because it allows for early rehabilitation, shortening surgery and hospitalization time, lowering treatment cost, and allowing the patient early psychosocial reintegration (14).

The use of implants in maxillofacial prosthetics provides patients with a predictable esthetics, improved retention and stability of their prostheses in comparison with other retention methods. Traditional methods include the use of medical-grade skin adhesives, spectacles, and tissue undercuts. These modalities are associated with difficulties related to retention reliability, stability, adverse tissue reactions, and accelerated discoloration and prosthesis deterioration, discomfort, and reduced acceptance(15).

Implant retained prostheses are a suitable option for their enhanced retentive property and are preferable to surgical reconstruction which may have unpredictable results. The construction of the missing auricle puts a test on the skill of the prosthodontist to reproduce the form, texture and tone of the existing contralateral ear but its successful rehabilitation is a rewarding experience for the dentist and patient alike (15).

Since the introduction of endosseous implants for use with bone conduction hearing aids in 1970s, the use of osseointegrated implants to retain facial prostheses has acquired an important role in the prosthetic rehabilitation of patients with craniofacial defects and became an integral part of treatment planning for facial reconstruction (16-17). Implant retention is currently considered as the gold standard in prosthetic reconstruction of these structures. The success of bone-anchored auricular prostheses could base upon the patients' acceptance, contribution to quality of life and use of the prostheses as replacement prosthesis for either a developmental defect or acquired defect $(18,19)$.

The use of cranial implants has also provided an alternative approach towards rehabilitating patients with severe auricular defects since 1977 (20) and has become a viable option that can offers several advantages over traditional reconstructive techniques. It has been suggested that, auricular implants enhance retention and stability of prostheses (21), improving the patient's confidence and sense of security. In addition (19), attachment systems aid in the proper positioning of prostheses, facilitating insertion by the individuals with auricular defects. The etiology of the loss of an auricle can be either acquired or congenital. Among acquired cases, gunshot injuries, traffic accidents etc, burns, ablative cancer surgeries are the reasons $(18,19$ and 22).

As a result, An implant-retained auricular prosthesis is also an option that can be considered instead of surgical reconstruction $(22,23)$. The application of craniofacial implants for facial disfigurement was first reported by Tjellström(24). The use of implants has an obvious advantage of better retention but then it is also associated with the potential surgical complications and cost related factors. Many implant systems are currently available for intraoral use, with different strategies and designs to match and overcome difficult oral conditions, improving the prosthetic outcome. However, craniofacial implants have not gained much attention, largely due to the small population of patients in need of extraoral rehabilitation and the few systems available $(25,26)$.

Most of the studies reported using the 2-stage surgical procedure for placing craniofacial implants, to allow for undisturbed healing (22, 23, 27-29). However, Khamis et al (30) described a new technique with modified abutments in implant-retained auricular prostheses, using a single- stage surgical procedure. They (30) have screwed the modified Oring abutments directly onto the implants at the time of surgery. Plastic washers were attached to the O-ring heads of the exposed abutments to avoid skin overgrowth to allow a single-stage surgical procedure. After an osseointegration period of 4 months, a silicone prosthetic ear was fabricated and retained using clips over the O-ring abutments. 


\section{MATERIALS AND METHODS}

Patients were informed about the treatment procedures and follow-up examinations. Informed consent was filled out of each patient in accordance with the regulation of Ethics Committee in Faculty of Dentistry, Alexandria University.

Ten patients age range, (6-33 years with a average mean of 20 years), presented with fourteen auriculars defects involved in this study. All the patients enrolled in the present study agreed to participate in it, provided with a confirmed consent, and then 42 implants placed among them. Patients that were enrolled in the study were selected of Adequate medical health, with either Unilateral or Bilateral auricular loss. Patients who were withdrawn in the study have been presented with either total or partial loss of auricle (Anotia or Microtia), and their ages were not to be less than 6 years old.

Only Four of the selected patients had congenital auricular defects ranging between microtia type I and II, while the rest six patients had an acquired ones resulted from accident. None of the selected patients had lost their ears due to malignant nor had undergone neither radiotherapy nor chemotherapy. In addition, four of the selected patients have bilateral auricular defects.

All the patients in the current study undergone a skin grafted single stage implant surgery with ball and socket attachment as a first stage treatment. Forty-two intra -oral implants (c-tech BL, Italy) were used for fourteen patients. Clinical Procedures were done using Pre-operative examination to assure case selection according to research diagnostic criteria of maxillofacial mastoid region examination. Planning method using CBCT of the mastoid region and clinical photographs of the patient were obtained preoperatively. Medical evaluation for patients before surgical intervention was made for surgical preparation. An irreversible hydrocolloid impression material ( Kromalgin, high precisions alginate , Italy) was made for the unaffected side if present which was obtained for the contra lateral ear and in case of bilateral loss of ears, a patient relative donation was made after the approval of both the patient and the donor.

A definitive impression was taken for the affected (absent ) auriclar side using additional silicone elastomeric impression material (ExamixTM , Monophase, GC, America Inc.) to be poured in type IV stone cast (extra-hard stone) were the wax model sculpture (figure 2) obtained from the contra lateral one was to match it in size and shape. In cases of bilateral loss, donor's ear should be modified to match patient's face and features. The Sculpted wax ear should be evaluated on the patient using facial guidelines.

The surgical guiding template (positioner) of clear acrylic resin was poured. Pre- planned six drilled holes were made using copper bands in the positioned, which corresponds precisely to the expected prosthetic implant position to guide in craniofacial implant placement. two holes were drilled for each implant in the positioner using No 12 round bur that made , the holes were filled with metal balls neighboring to each other with same inclination on the same horizontal line to allow the choice of the proper implant position hole using the CBCT super imposition. For each implant one hole was picked out of the two holes and tabulated for surgical drilling. Evaluation of the CBCT with the surgical template to choose the most suitable of the drilled holes as regards the adequate bone thickness.
The titanium implant (C-tech. BL type) (figure 1) was provided in $6 \mathrm{~mm}$ length with a standard diameter of 4.8 $\mathrm{mm}$, a 2-mm height of smooth collar, a reverse buttress thread, a flat platform, and an internal hex abutment interface. It was placed in the mastoid region of a bone thickness averaged from 5 or $6 \mathrm{~mm}$ in this area as a recipient site for implant retained auricular epistheses (non-irritated predictable area for placement). Patients undergone general anesthetic surgery to place the implants with a curvy linear post auricular incision. All the cases had received implants of $6 \mathrm{~mm}$ height and $4.8 \mathrm{~mm}$ in diameter.

A clear acrylic resin surgical template (positioner) was placed in its accurate position according to the proper location with the ledge extension in the external meatus located by the CBCT to allow for implant drilling; where the implant should be placed in the chosen hole $20 \mathrm{~mm}$ away from the external acoustic meatus and $15 \mathrm{~mm}$ from each other. Skin and subcutaneous tissues were reflected using a full thickness incision flap with undermined margins where the bone surface was exposed.

The implants were inserted by drilling using standard surgical kit (c-tech implant kit) at the sites that were marked with surgical template (positioner). The surgical kit included four drills in addition to a pilot drill, which was used as a primary drill followed by the other four drills until the final one that corresponds to the specific implant diameter $(4.8 \mathrm{~mm})$. They were inserted parallel to each other under minimal trauma to prevent heat injury to the surrounding bone and to ensure primary osseointegration. A finger wrench was used for initial screwing of the implants followed by the ratchet wrench (20-35 N/cm) which indicate initial stabilization and strong anchorage within bone.

After the implants were placed, the healing abutments were directly screwed into the implant manually and tightened according to the amount of the skin graft, where their heights ranged from 2 to $6 \mathrm{~mm}$ according to the autogenous skin (auto-donated) graft thickness was used in a single stage procedure. The graft was placed to cover the implant collar.

Perforations were made in the flap corresponding to the implants positions; afterwards the flap was sutured in place using non-resorbable suture (proline-6/0) then covered with ointment-soaked gauze to protect the skin.

The sutures were removed one week after the surgery, and the patient was asked to apply antibacterial ointment once or twice a week for the first three months. Small self-cured acrylic resin buttons were shaped around each healing abutment, to avoid surgical edema, swelling and skin growth around the abutments. The patients were left for 3- 4 month after surgery then was evaluated for prosthetic management using Prosthetic material A-2000 Silicon Elastomer, Factor II, Incorporated, A-2000 Silicone Elastomer product Lakeside, AZ USA).

At the end of the recommended period of 3-4 months for osseointegration, the implant /abutment was evaluated for the periods of 1,4, 6 and 12 months, where The criteria for determining successful osseointegration included: Mobility test for implants osseointegration

Absence or Presence of clinically detectable implant mobility was checked by applying back and forth pressure using the back of two hand instruments on the sides of implant abutment. Grade 0: No mobility, Grade 1: mild mobility, Grade 2: Moderate mobility and Grade 3: 
Aggressive mobility (failure to integrate). Absence or presence of spontaneous pain or pain during abutment tightened was assessed clinically by the patient.

Peri-implant abutment tissue visual examination was monitored to assess inflammation level by Gitto et al. (31) giving a scale from 0-4, were Grade 0: Normal skin ,Grade 1:Mild inflammation (slight redness and/or edema), Grade 2: Moderate inflammation (redness, edema, mild tenderness) and Grade 3: Severe inflammation (marked redness, edema, ulceration, moderate to severe pain).

Peri-implant abutment exudates secretion around implant abutment connection was done by the visual inspection of exudates was monitored through the four periodic evaluation of osseointegration (1,4,6 and 12 months) as the criteria done by Gitto et al.(31) Grade 0: No exudate, Grade 1: Serous exudate and Grade 2: Purulent exudate.

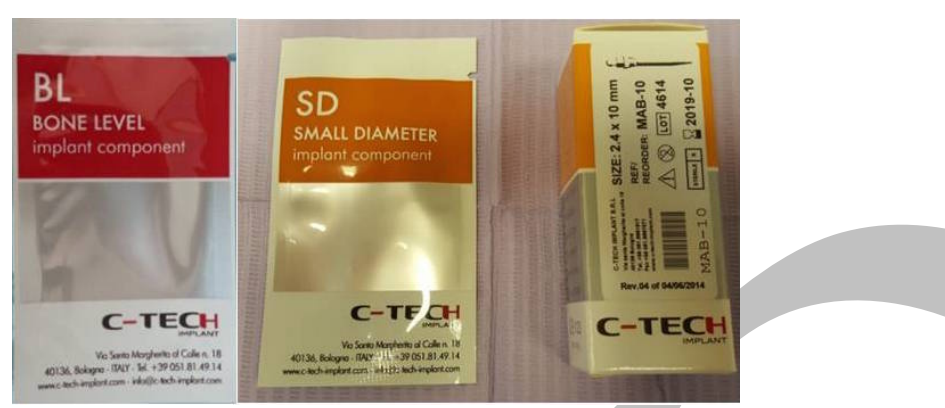

Figure 1: Intraoral implant(c-tech BL, Italy).
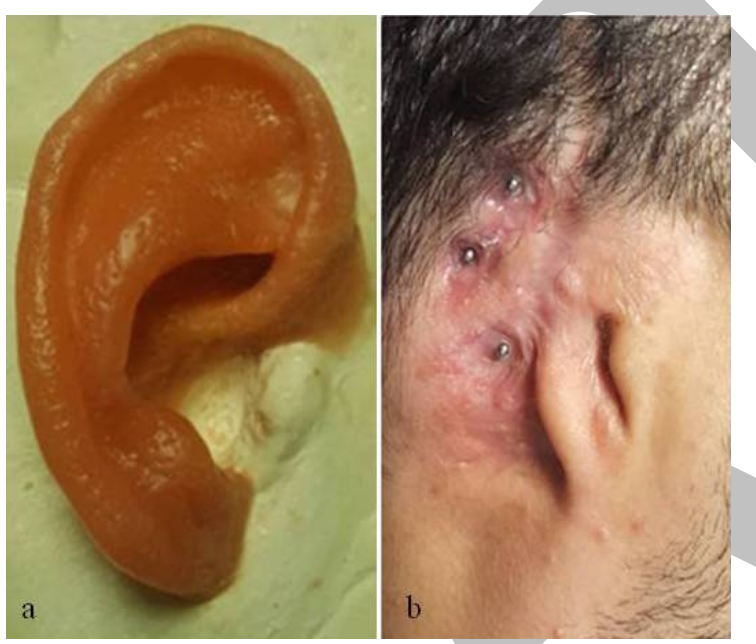

Figure 2: A .Wax sculpted ear, b. Ball and socket attachment implant abutment

Skin crusting around implant/abutment connection was examined by visual inspection of the skin around the implant abutment connection , this provides the evaluation of patient hygiene by the use of four grades that were included in the criteria that was proposed by Gitto et al.(31) Grade 0 : No crusting, Grade 1: Small amount of crusting but not encircling the entire abutment, Grade 2: Moderate amount of crusting not encircling the entire abutment, Grade 3: Heavy accumulation of crusting encircling the entire abutment.

skin thickness (probing depth) has been evaluated by using plastic periodontal prob that was inserted with the long axis of the implant on four different points around the implant abutment connection. Readings were tabulated and average measured in which abutment height was chosen in accordance or being replaced.

\section{STATISTICAL ANALYSIS}

The date was collected, tabulated and statistically analyzed by using descriptive data analysis by calculating the mean and standard deviation.

\section{RESULTS}

In the present study, 42 implants were placed in 14 auricular defects involving total of ten patients (4 patients with bilateral auricular defects).

Evaluation of the peri-implant abutment tissues reaction values obtained following the index described by Gitto et al (31) at different time intervals of 1 month, 3 months, 6 months and 12 months, (table I). At 1 month interval, $35.71 \%$ of the patients showed normal overlying periimplant tissues, whereas $42.85 \%$ showed mild non-tender inflamed tissues with slight redness (score=1). Only 21.42\% of patients showed moderately inflamed and reddened tissues with slight tenderness (score=2). None of them showed severe inflammation with marked redness, edema, ulceration and moderate to severe pain. The percent of patients showing score $=1$ and score $=2$ decreased to $14.29 \%$ and $7.14 \%$ at 3 months respectively, while at 6 and 12 months only 1 patient remained to show mild inflammatory tissue reaction of score $=1$. The mean Value of tissue reaction showed a decrease across the time periods from 1 month to 12 months recall (figure 3).

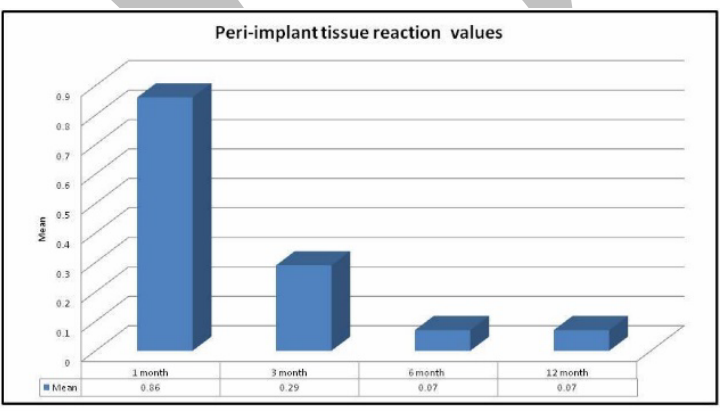

Figure 3: Bar graph showing the mean of tissue reaction at 1, 3, 6 and 12 months recall.

Skin thickness values were evaluated in mm. upon different time intervals of 1 month, 4 months and 12 months $(n=14)$ as shown in (table II). The results of measurement of skin thickness values showed an overall decrease in the mean across the time intervals from 1 to 12 months recall, At 1-month recall, the skin thickness ranged between $2-8$ $\mathrm{mm}$, on the other hand this range decreased at 4 months and 6 months recalls (figure 4 ).

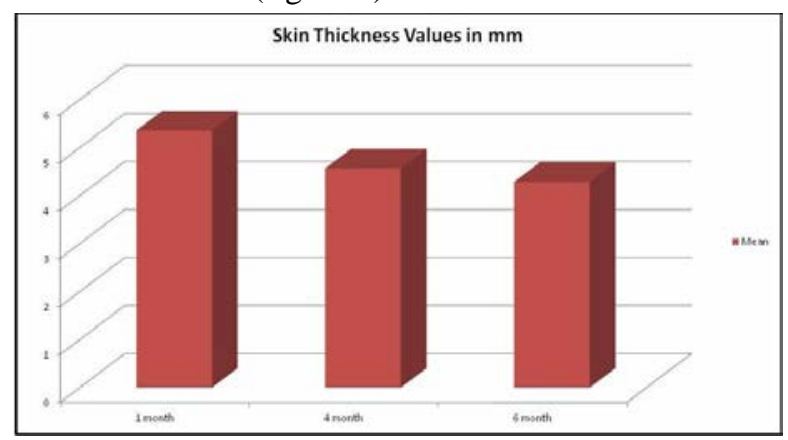

Figure 4: Bar graph showing mean value of skin thickness at 1, 4 and 6 months recall.

Average sebaceous crusting values guided by the index described by Gitto et al (31) at different recalls of 1 month, 4 months and 6 months were evaluated, (Table III). At 1 
month recall, $50 \%$ of patients (7 out of 14 ) showed small amount of crusting not encircling the entire abutment (grade 1), moreover $35.7 \%$ (5 out of 14 ) showed moderate amount of crusting not encircling the entire abutment (grade 2), while $14.3 \%$ of patients (2 out of 14) showed heavy accumulation of crusting encircling the entire abutment.

At 4 months interval, $42.9 \%$ of patients presented with no crusting, with a decrease in the no. of patients presenting with grade 1, 2 and 3 crusting with percent 28.6\%, 21.4\% and $7.1 \%$ respectively. The percent of patient with grade 0 increased to $50 \%$ at the 6 month recall. In addition, none of them presented with grade 3 crusting (figure 5).

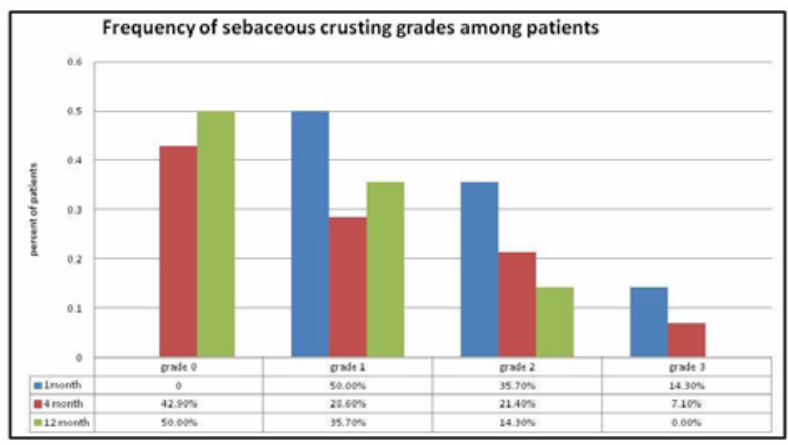

Figure 5: Bar graph representing the frequency of sebaceous crusting grades recall.

The average of sebaceous crusting showed a range and mean of at one and 6 months recall and 3 at 4 months recall. The mean value of crusting was noticed to be $1.64+0.745$, $0.93+0.997$ and $0.64+0.745$ at one , 4 and 6 months, indicating an improvement in tissue healing .

Evaluation of peri-implant exudates values guided by the index described by Gitto et al (31) at different recalls of 1 month, 4 months and 6 months, (table IV). The results of peri-implant abutment values showed mean of $0.64+0.633$ at one month, $0.14+0.363$ at 4 month and $0.00+0.00$, where none of the patient showed exudates discharge at 6 months interval (figure 5). At one-month recall, 42.9\% showed no exudates (grade 0 ), $50 \%$ showed serous exudates (grade 1), and $7.1 \%$ showed purulent exudates (grade 2). At 4 month recall $85.7 \%$ showed no exudates and $14.3 \%$ presented with serous exudates. None of the patients presented with purulent discharge at this phase. At 6 months recall, none of the patients presented with exudates discharge whether serous or purulent (figure 6).

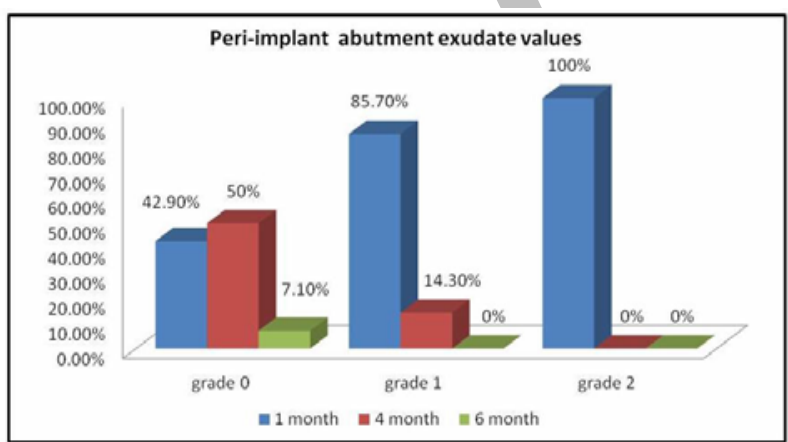

Figure 6: Bar graph showing peri-implant abutment exudates values at 1,4 and 6 months intervals.

\section{DISCUSSION}

Reconstruction of maxillofacial defects are considered a complex modality and requires a good cooperation in diagnosis and treatment planning based on the presence of two line of treatments (surgical and prosthetic) which can be solitary or in conjunction together.

Since the concept of osseointegration is similar whether implants are placed intraoral or exraoral, of intraoral implants were used in the present study as a mean of retention to replace the extraoral ones in the auricular region where they showed a great success and survival rate reaching up to 3 years in function. In the current study ; $6 \mathrm{~mm}$ height implant with $4.8 \mathrm{~mm}$ diameter and 2-mm height smooth collar were placed. They had a design of reverse buttress thread, a flat platform, and an internal hex abutment interface.

Apart of many advantages provided by the implants, the significant improvement in prosthesis retention without using adhesive has great added to patient acceptance. Granström et al (32) reported their experience of osseointegrated implant in 100 pediatric patients. The implant failures were $5.8 \%$ of 170 inserted fixtures. In another study, Korus et al (33) assessed the long-term outcomes of osseointegrated ear reconstruction procedures performed on 69 pediatric and adult patients. The results of that study showed that patients were generally satisfied with the osseointegrated implant.

A post surgical radiographic evaluation of implant osseointegration in the mastoid region was done using a CBCT showed a complete integration success with no evidence of any discontinuity in implant bone interface. This finding was coherent with multiple studies (32,34,35).

During the follow up visits along the three years none of the implant abutments have shown an evidence of mobility or abutment loosening clinically . Abutments were checked using a hand wrench to avoid unnecessary torque or pressure applied to any. This finding is in agreement with Nishimura et al.(34) and Wrigh et al. (35) have reported a high survival rate and life span of implant retained auricular prosthesis as well as karakoca et al. (19) has reported similar results.

Three implants have been placed in each auricular defect as to allow for proper retention and more secured attachment, as well as giving patient good predictability of placing it in the proper direction. Although some researchers (36) have recommended that 2 implants are enough for function, because the episthesis is not heavy, others (34) have preferred 3 or 4 implants when the computerized tomography sections were examined and the temporal bone quality seemed to be appropriate for osteointegration. In this case report 3 implants were used because of the temporal bone quality.

It has been known that implant retained auricular prosthetic surgeries may be performed in a single or two stage surgery. In the current study, all 42 implants were done using single stage surgery that was preferred over the two stage one as to avoid the scar tissue formation resulting from surgery and relief the patient from the fear and expenses of going through two general anesthetic surgery. Also, Sencimen et al. (37) also stated that the two-stage procedure is not recommended so as not to compromise the vascular supply of the area.

Once implants are successfully placed, 3 basic retentive systems can be used: the bar and clip, the ball and keeper, and the magnet and keeper. The bars and clips, in some studies (38), showed disadvantages of being of high cost 
and the increased difficulty for the patient in performing the necessary implant-associated hygiene. Therefore, the use of ball and socket attachment preferred over bar-clip attachment in the present study to allow the ease of manipulation, use and placement of the prosthesis by the patient as well as the prosthodontist. As this finding is in agreement with karakoca et al. (19) who stated that the most frequent complications are mechanical failure including broken or lost clips, clip fracure, and frequent need of clip activation using the clip activator, which are done by the Prosthodontist.

Implant success and peri-implant tissue health were evaluated clinically as it has been found to be the most feasible method for extraoral implant evaluation. Unlike intraoral implants, periodic radiographic monitoring of craniofacial implants was found not to be practical (31).

$35 \%$ of patients have shown no signs of pain or redness in the pericuenous issues around implants, where $43 \%$ showed mild redness with no evidence of pain, giving only $21 \%$ who suffered from moderate inflammation and redness that has been improved significantly over time. None of the patients showed severe inflammation with marked redness, edema, ulceration.

Therefore, the absence of adverse and noticeable reaction in the percutenous tissues around implants clinically in the present study was found to be likely evidenced by Tjellsrom et al.(24) and Nishimura et al. (34) who mentioned that the frequency of adverse skin reactions around the soft tissues of the percutaneous implant is generally very low and the main symptomatic reactions may consists of slight redness, moistened peri-implant tissues. The likelihood of losing an implant because of adverse skin reactions is quite low, but if these skin reactions are left untreated, implant loss or withdrawal may eventually occur (39).

$50 \%$ of patients (7 out of 14) showed small amount of crusting not encircling the entire abutment moreover 35.7\% showed moderate amount of crusting not encircling the entire abutment, while $14.3 \%$ of patients ( 2 out of 14 ) showed heavy accumulation of crusting encircling the entire abutment.

The presence of slight crusting around implant abutment was found mostly in the first month showing a noticeable decrease during recall interval visits as patients were being instructed with a daily regime (protocol) and ointment placement post surgical to allow for minimizing pain, redness and allowing edema to subside. Also Gumieiro et al (39) have stated that good patient hygiene compliance results in minimal soft tissue complications.

Patients who have been manifested with heavy accumulation of crusting (2 out of 14), have been instructed with a strict hygiene protocol and been more frequently recalled for follow up. This finding was noticed among young age group patients less than 10 years that may be attributed to age mental level as well as socioeconomic status.

Skin thickness around implant abutment connection in the study has shown a range of 2-8 mm. Most of the cases who presented with $8 \mathrm{~mm}$ skin thickness 1 month postsurgical were found to be of young age group (below 16 years old) while in older age group it was found to be of lesser skin thickness values and that corresponds to higher skin proliferation activity. This finding is in agreement with Tolman et al (32). In addition, patient who suffered from burn accidents, showed minimal (least) levels of skin thickness.This was a result of the diminished blood supply to the skin grafted site.

Skin thickness around implant abutment connection has been significantly changed over the given recall intervals but it has shown improvement and decreased in the thickness in the 3 and 6-month recall visits giving the explanation of edema resolution after surgery. It was found that, both hygiene as well as ointment medications helped the resolving of edema. This finding was in accordance with Gumieiro et al. (39). Studies (1, 31and 34) have reported that patient with worst skin reactions has manifested thick peri-implant issues and thickness will result in increasing complications.

At one-month recall, half of the patient showed serous exudates secretion that was noticeably improved over time, where it was found at 6 months recall visit that none of the patients presented with exudates discharge whether serous or purulent. Those results matched the results of pericutaneous implant tissue reaction values which corresponded to patient level of compliance to hygiene measures (40) .

Finally, osseointegrated prosthesis reconstruction is a long-term commitment of both patient and prosthetic team is required to allow optimum results. , therefore reliability and compliance of the patient as well as, the availability of a multidisciplinary team of caregivers are needed for the success of this procedure. It should also be always noted that the prosthesis is not one's own tissue.

Table I: Peri-implant abutment tissues reaction values.

\begin{tabular}{c|c|c|c|c}
\hline \multicolumn{5}{c}{ Peri-implant abutment tissues reaction values } \\
\hline Defect & 1 month & 3 months & 6 months & 12 months \\
\hline 1 & 0 & 0 & 0 & 0 \\
\hline 2 & 1 & 0 & 0 & 0 \\
\hline 3 & 1 & 0 & 0 & 0 \\
\hline 4 & 2 & 1 & 0 & 0 \\
\hline 5 & 0 & 0 & 0 & 0 \\
\hline 6 & 1 & 0 & 0 & 0 \\
\hline 7 & 1 & 0 & 0 & 0 \\
\hline 8 & 0 & 0 & 0 & 0 \\
\hline 9 & 1 & 0 & 0 & 0 \\
\hline 10 & 1 & 0 & 0 & 0 \\
\hline 11 & 0 & 0 & 0 & 1 \\
\hline 12 & 2 & 2 & 1 & 0 \\
\hline 13 & 0 & 0 & 0 & 0.267 \\
\hline 14 & 2 & 1 & 0 & 1 \\
\hline Range & 2 & 2 & 0.07 & 0.267 \\
\hline Mean & 0.86 & 0.29 & & 0 \\
\hline Standard Deviation & 0.770 & 0.611 & & 0 \\
\hline
\end{tabular}


Table II: Skin thickness values in mm.

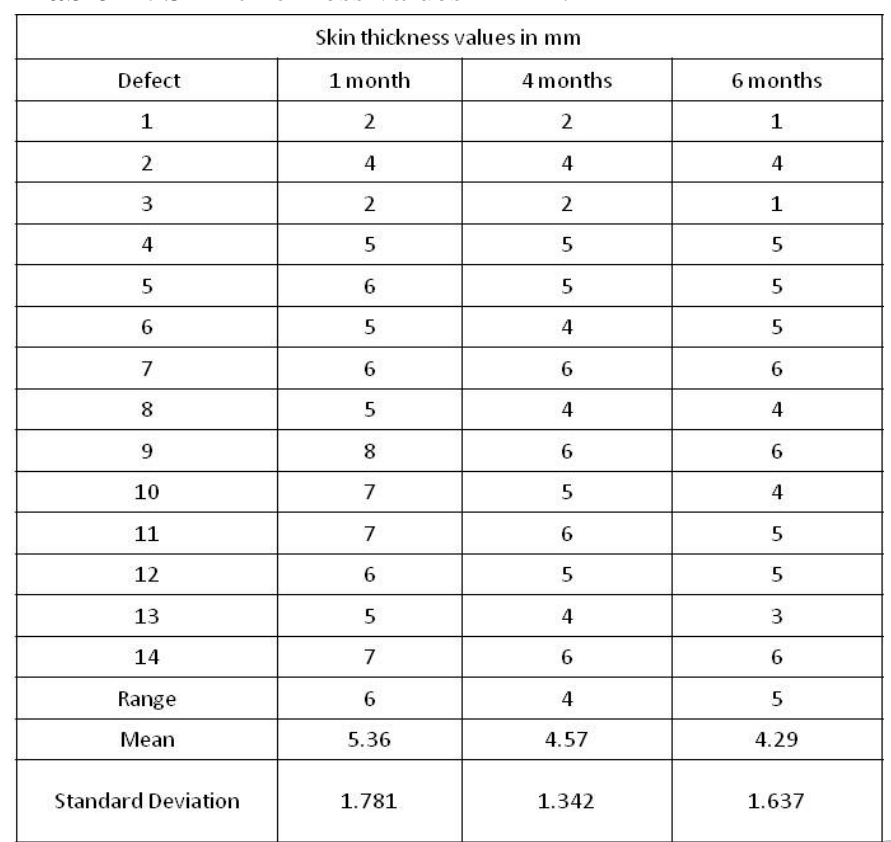

Table III: Average of sebaceous crusting.

\begin{tabular}{|c|c|c|c|}
\hline \multicolumn{4}{|c|}{ Average of sebaceous crusting } \\
\hline Defect & 1 month & 4 months & 6 months \\
\hline 1 & 2 & 2 & 1 \\
\hline 2 & 1 & 0 & 0 \\
\hline 3 & 1 & 1 & 0 \\
\hline 4 & 2 & 2 & 1 \\
\hline 5 & 1 & 0 & 0 \\
\hline 6 & 1 & 0 & 0 \\
\hline 7 & 3 & 1 & 1 \\
\hline 8 & 2 & 2 & 2 \\
\hline 9 & 2 & 1 & 1 \\
\hline 10 & 1 & 0 & 0 \\
\hline 11 & 1 & 0 & 0 \\
\hline 12 & 1 & 0 & 0 \\
\hline 13 & 2 & 1 & 1 \\
\hline 14 & 3 & 3 & 2 \\
\hline Range & 2 & 3 & 2 \\
\hline Mean & 1.64 & 0.93 & 0.64 \\
\hline Standard Deviation & 0.745 & 0.997 & 0.745 \\
\hline Variance & 0.555 & 0.995 & 0.555 \\
\hline
\end{tabular}

\section{REFERENCES}

1. Beumer J, Curtis TA, Firtell DN. Maxillofacial prosthetics: Surgical and Prosthodontic Considerations, St. Louis: Mosby, 1979.

2. Semple C, Parahoo K, Norman A, McCaughan E, Humphris G, Mills M. Psychosocial interventions for patients with head and neck cancer. Cochrane Database of Systematic Reviews 2013: 41-94.

3. Mantri S and Khan Z. Prosthodontic Rehabilitation of Acquired Maxillofacial Defects. Head and Neck Cancer Rijeka: InTech; 2012: 315 - 36.

4. Jaffe B. The incidence of ear diseases in the Navajo Indians. Laryngoscope. 1968;79:2126-34.

5. Luquetti DV, Heike CL, Hing AV, Cunningham ML, Cox TC. Microtia: Epidemiology and genetics. Am J Med Genet A. 2011;158A:124-39.

6. Horlock N, Vögelin E, Bradbury ET, Grobbelaar AO, Gault DT. Psychosocial outcome of patients after ear 
reconstruction: A retrospective study of 62 patients. Ann Plast Surg. 2005;54:517-24.

7. Marx H. Die Missbildungen des ohres. In: Denker AK, editor. Handbuch der Spez Path Anatomie Histologie. Berlin: Springer; 1926:131.

8. Meurman Y. Congenital microtia and meatal atresia; observations and aspects of treatment. AMA Arch Otolaryngol. 1957;66:443-63.

9. Walton RL, Beahm EK. Auricular reconstruction for microtia: Part I Surgical techniques. Plast Reconstr Surg. 2002;110:234-49.

10. Tanzer RC. Total reconstruction of the external ear. Plast Reconstr Surg Transplant Bull. 1959;23:1-15.

11. Brent B. Microtia repair with rib cartilage grafts: A review of personal experience with 1000 cases. Clin Plast Surg. 2002;29:257-71.

12. Reinisch JF, Lewin S. Ear reconstruction using a porous polyethylene framework and temporoparietal fascia flap. Facial Plast Surg. 2009;25:181-9.

13. Brånemark B, Brånemark PI, Rydevik B, Myers RR. Osseointegration in skeletal reconstruction and rehabilitation: A review. J Rehabil Res Dev. 2001;38:17581.

14. Hatamleh MM, Watson J. Construction of an implantretained auricular prosthesis with the aid of contemporary digital technologies: A clinical report. J Prosthodont. 2013;22:132-6.

15. Hatamleh MM, Haylock C, Watson J, Watts DC. Maxillofacial prosthetic rehabilitation in the UK: A survey of maxillofacial prosthetists' and technologists' attitudes and opinions. Int J Oral Maxillofac Surg. 2010;39:118692.

16. Granström G. Craniofacial osseointegration. Oral Dis. 2007;13:261-9.

17. Scolozzi P. , Jaques B. Treatment of mid-facial defect using prostheses supported b ITI dental implants. Plast Reconstr surg. 2004;114:1359-404.

18. Karayazgan-Saracoglu, B.; Zulfikar, H.; Atay, A.\& Gunay, Y. Treatment outcome of extraoral implants in the craniofacial region. J Craniofac Surg. 2010; 21:751-758.

19. Karakoca, S., Aydin, C., Yilmaz, H.\& Bal, B.T. Retrospective study of treatment outcomes with implant-retained extraoral prostheses: survival rates and prosthetic complications. J Prosthet Dent. 2010; 103:118126.

20. Niparko JK, Langman AW, Cutler DS, Carroll WR. Tissue-integrated prostheses in the rehabilitation of auricular defects: results with percutaneous mastoid implants. Am J Otol. 1993;14:343-8.

21. Miles BA, Sinn DP, Gion GG. Experience with cranial implant-based prosthetic reconstruction. J Craniofac Surg. 2006;17:889-97.

22. Tjellström A. Osseointegrated implants for replacement of absent or defective ears. Clin Plast Surg 1990;17:355-66.

23. Parel SM, Holt GR, Bränemark PI, Tjellström A. Osseointegration andfacial prosthetics. Int $\mathrm{J}$ Oral Maxillofac Implants 1986;1:27-9.

24. Tjellström A, Yontchev E, Lindström J, Bränemark PI. Five years' experience with bone-anchored auricular prostheses. Otolaryngol Head Neck Surg 1985;93:366-72.

25. Thomas KF. Freestanding magnetic retention for extraoral prosthesis with osseointegrated implants. J Prosthet Dent 1995;73:162-5.
26. Reisberg DJ, Habakuk SW. Hygiene procedures for implant-retained facial prostheses.J Prosthet Dent 1995;74:499-502.

27. Lundgren S, Moy PK, Beumer J 3rd, Lewis S. Surgical considerations for endosseous implant in the craniofacial region: a 3-year report. Int J Oral Maxillofac Surg 1993;22:272-7.

28. Seals RR Jr, Cortes AL, Parel SM. Fabrication of facial prostheses by applying the osseointegration concept for retention. J Prosthet Dent 1989;61:712-6.

29. Arcuri MR, LaVelle WE, Fyler A, Funk G. Effects of implant anchorage on midface prostheses. J Prosthet Dent 1997;78:496-500.

30. Khamis, M.M., Medra, A. \& Gauld, J. Clinical evaluation of a newly designed singlestage craniofacial implant: a pilot study. J Prosthet Dent. 2008;100:375-383.

31. Gitto CA, Plata WG, Schaaf NG. Evaluation of the periimplant epithelial tissue of percutaneous implant abutments supporting maxillofacial prostheses. Int J Oral Maxillofac Implants 1994;9:197-206.

32. Granström G, Bergström K, Odersjö M, Tjellström A. Osseointegrated implants in children: Experience from our first 100 patients. Otolaryngol Head Neck Surg. 2001;125:85-92.

33. Korus LJ, Wong JN, Wilkes GH. Long-term follow-up of osseointegrated auricular reconstruction. Plast Reconstr Surg. 2011;127:630-6.

34. Nishimura RD, Roumanas E, Moy PK, Sugai T, Freymiller EG. Osseointegrated implants and orbital defects: U.C.L.A. experience. J Prosthet Dent, 1998;79:304-9.

35. Wright, R.F.; Zemnick, C.; Wazen, J.J. \& Asher, E. Osseointegrated implants and auricular defects: a case series study. J Prosthodont. 2008;17 :468-475.

36. Wazen JJ, Wright R, Hatfield RB, Asher ES. Auricular rehabilitation with bone anchored titanium implants. Laryngoscope. 1999;109:523-527.

37. Sencimen M., Bal H.E., Demirogullari M., Kocaoglu M., Dogan N. Auricular episthesis retained b an attachment system (2 case reports). Oral Surg Oral Med Oral Pathol Oral Radiol Endod, 2008;105:28-34.

38. Del Valle V, Faulkner G, Wolfaardt J, Rangert B, Tan HK. Mechanical evaluation of craniofacial osseointegration retention systems. Int J Oral Maxillofac Implants. 1995;10:491-498.

39. Roumanas E, Nishimura R, Beumer J III, Moy P, Weinlander $\mathrm{M}$, Lorant J. Craniofacial defects and osseointegrated implants: six-year follow-up report on the success rates of craniofacial implants at UCLA. Int J Oral Maxillofac Implants 1994;9:579-85.

40. Gumieiro E.H., Luciano L.D., Jahn R.S., Ferreira J., Granstrom N.U. \& Abrahao G. M. Bone-anchored titanium implants for auricular rehabilitation: case report and review of literature. Sao Paulo Med J 2009;127: 1605 . 